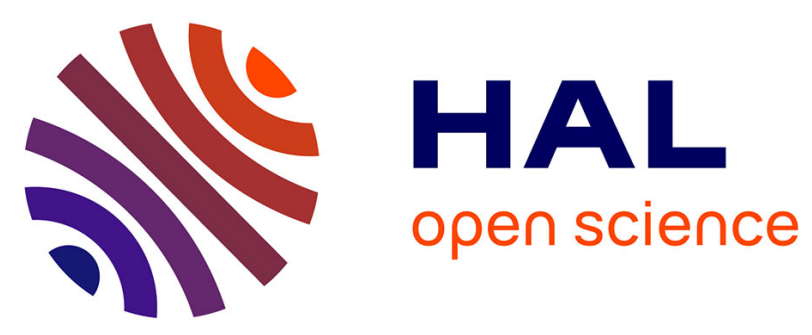

\title{
Adaptive Acquisitions In Biomedical Optical Imaging Based on Single pixel Camera: Comparison With Compressive Sensing
}

Florian Rousset, Nicolas Ducros, Andrea Farina, Gianluca Valentini, Cosimo d'Andrea, Françoise Peyrin

\section{To cite this version:}

Florian Rousset, Nicolas Ducros, Andrea Farina, Gianluca Valentini, Cosimo d'Andrea, et al.. Adaptive Acquisitions In Biomedical Optical Imaging Based on Single pixel Camera: Comparison With Compressive Sensing. International Symposium on Biomedical Imaging: From Nano to Macro (ISBI 2016), Apr 2016, Prague, Czech Republic. hal-01288056

\section{HAL Id: hal-01288056 https://hal.science/hal-01288056}

Submitted on 29 Apr 2016

HAL is a multi-disciplinary open access archive for the deposit and dissemination of scientific research documents, whether they are published or not. The documents may come from teaching and research institutions in France or abroad, or from public or private research centers.
L'archive ouverte pluridisciplinaire HAL, est destinée au dépôt et à la diffusion de documents scientifiques de niveau recherche, publiés ou non, émanant des établissements d'enseignement et de recherche français ou étrangers, des laboratoires publics ou privés. 


\title{
ADAPTIVE ACQUISITIONS IN BIOMEDICAL OPTICAL IMAGING BASED ON SINGLE PIXEL CAMERA: COMPARISON WITH COMPRESSIVE SENSING
}

\author{
Florian Rousset $^{1,2}$, Nicolas Ducros ${ }^{1}$, Andrea Farina ${ }^{3}$, Gianluca Valentini ${ }^{2}$ \\ Cosimo D'Andrea ${ }^{2,4}$, Françoise Peyrin ${ }^{1}$ \\ ${ }^{1}$ Université de Lyon, CREATIS ; CNRS UMR5220 ; Inserm U1206 ; INSA-Lyon \\ Université Claude Bernard Lyon 1 - Villeurbanne, France \\ ${ }^{2}$ Politecnico di Milano, Dipartimento di Fisica - Milano, Italy \\ ${ }^{3}$ Consiglio Nazionale delle Ricerche, IFN - Milano, Italy \\ ${ }^{4}$ Center for Nano Science and Technology@PoliMi, Istituto Italiano di Tecnologia - Milano, Italy
}

\begin{abstract}
Single pixel imaging opened the door to a cheaper camera architecture able to operate in a wide spectral range. Such an optical setup has been used with compressed sensing to reconstruct an image via $\ell_{1}$-minimization ruling out real time applications. In order to have a direct restoration of the image, we consider an adaptive approach for which we propose a new acquisition strategy. Our method progressively acquires an image in the wavelet domain by predicting the significant coefficients. For this, we base our technique on the non-linear approximation of the wavelet transform taking advantage of the transformation's sparsity. This new strategy is shown to offer high performance on simulated and real data that we compare to compressive sensing acquisitions. One possible application of the single pixel camera can be foreseen in fluorescence images of biological structures.
\end{abstract}

Index Terms - Single-pixel camera, compressed sensing, wavelet transform, image processing

\section{INTRODUCTION}

Building small, cheap and efficient sensors becomes possible when considering the single pixel camera (SPC) architecture [1]. Several advantages can be noted compared to CCD or CMOS cameras. First, building a unique sensor for infrared or hyperspectral imaging considerably reduces the costs with regard to a conventional camera operating at these wavelengths. Second, the single detector can have a very good quantum efficiency and few storage memory is needed. Finally, SPC is also well suited to create a low-cost timeresolved imaging device by using a single TCSPC (TimeCorrelated Single Photon Counting) board.

Our goal in this paper is to provide a new acquisition strategy for SPC acquisitions. The single pixel camera can find many applications in the biomedical field. For instance, infrared imaging can be considered to study collagen lipid interaction, hyperspectral imaging for tissue oxygenation or time-resolved imaging for fluorescence lifetime imaging [2].

\section{PROBLEM AND RELATED WORK}

We address the problem of recovering the image of an object acquired by a SPC, which was originally formulated in [1, 3]. The optical setup can be implemented with a digital micromirror device (DMD) and a single detector element. A lens is added to focus light on the single detector. A DMD has thousands of mirrors that can be independently tilted in two states. The ON state reflects the light toward the detector whereas the OFF state reflects the light in the opposite direction. Hence, a DMD can act as a tunable spatial filtering device.

A SPC acquisition can be formalized as the sequential measurements of the dot product of the image and some DMD patterns. Let $\mathbf{F} \in \mathbb{R}^{N \times N}$ be the $N \times N$ image and $\mathbf{f} \in \mathbb{R}^{P \times 1}$ denote its vectorized form with $P=N^{2}$. Let $\left\{\mathbf{p}_{i} \in \mathbb{R}^{P \times 1}, i=\right.$ $1 . . I\}$, a sequence of $I$ DMD patterns. The measurements $\left\{m_{i}, i=1 . . I\right\}$ can be expressed as

$$
m_{i}=\left\langle\mathbf{f}, \mathbf{p}_{i}\right\rangle
$$

Then, the problem consists in retrieving $\mathbf{f}$ from $\left\{m_{i}\right\}$, knowing the patterns $\left\{\mathbf{p}_{i}\right\}$.

Two main approaches exist: nonadaptive and adaptive. For the first type, the authors in [1,3] used compressed sensing [4]. Random patterns are considered and $\mathbf{f}$ is restored via $\ell_{1}$-minimization. For the second approach, the patterns are progressively determined during the acquisition $[5,6,7]$.

In this paper, we consider an adaptive scheme since it improves the image restoration without the computational overhead of $\ell_{1}$-minimization. Real time applications can thus be considered. In this paper, we obtain $\left\{m_{i}\right\}$ from wavelet patterns $\left\{\mathbf{p}_{i}\right\}$ using a non-linear acquisition strategy. In order to compare our method with the state-of-the-art compressive sensing (CS), we briefly introduce CS for SPC acquisitions 
in 3.1 before detailing our method in 3.3 that we refer to as Adaptive Basis Scan (ABS).

\section{METHODS}

\subsection{Compressive sensing for SPC}

Let $\mathbf{s} \in \mathbb{R}^{P \times 1}$ represents the signal $\mathbf{f}$ in the $\Psi$ domain where $\Psi \in \mathbb{R}^{P \times P}$ is an orthonormal transform operator such that

$$
\mathbf{f}=\Psi \mathbf{s}
$$

$\Psi$ can be chosen as a wavelet basis, Fourier basis, DCT basis, etc. We search a representation of $\mathbf{f}$ that is $K$-sparse i.e. only $K$ entries of $\mathbf{s}$ are non-zeros values.

Instead of acquiring $P$ measurements, the compressive sensing [4] aim to only retain $M<<P$ measurements as $y_{j}=\left\langle\mathbf{f}, \phi_{j}\right\rangle=\phi_{j}^{\top} \mathbf{f}$ with $M \approx K .\left\{\phi_{j}\right\}_{j=1}^{M}$ are referred to as measurement vectors and their transposed version can be arranged as rows of the sensing matrix $\Phi \in \mathbb{R}^{M \times P}$. Putting the measurement in the vector $\mathbf{y}$ and using (2), we can write

$$
\mathbf{y}=\Phi \mathbf{f}=\Phi \Psi \mathbf{s}=\Omega \mathbf{s}
$$

$\Omega$ is an $M \times P$ matrix and is completely independent from the image $\mathbf{F}$.

A common choice for the entries of the matrix $\Phi$ are independent realizations of \pm 1 Bernoulli random variables [1] with probability $1 / 2$. This sensing matrix is well suited for the DMD's ON/OFF states.

$\ell_{1}$-minimization is then employed to resolve the following problem:

$$
\hat{\mathbf{s}}=\arg \min \|\mathbf{s}\|_{1} \quad \text { such that } \Omega \mathbf{s}=\mathbf{y}
$$

Finally, one can recover the reconstructed signal by $\hat{\mathbf{f}}=\Psi \hat{\mathbf{s}}$ that can be reshaped into a $N \times N$ matrix to obtain the restored image $\hat{\mathbf{F}}$.

\subsection{Wavelet decomposition}

The discrete wavelet decomposition of an image with the standard dyadic separable wavelets transforms the image into approximation and detail coefficients (horizontal, vertical and diagonal). A low-pass filtering is applied to obtain the approximation image whereas the detail coefficients result from a high-pass filtering [8]. Let $j=1 \ldots J$ be the scale at which the image $\mathbf{f}$ is observed, $J$ being the decomposition level with $1 \leq J \leq \log _{2}(N)=R$. Let $k=\left(k_{1}, k_{2}\right) \in \mathbb{Z}^{2}$ specify a location, we note $\beta_{j, k}^{e}$ the coefficients, $e=0,1,2$ or 3 representing the approximation, horizontal, vertical and diagonal coefficients, respectively. These elements can be obtained by

$$
\beta_{j, k}^{e}=\left\langle\mathbf{f}, \gamma_{j, k}^{e}\right\rangle
$$

with $\gamma_{j, k}^{e}$ chosen as the scaling function $(e=0)$ or wavelet function ( $e=1,2$ or 3). Equations (1) and (5) show that the wavelet coefficients can be computed by the SPC.

\subsection{Adaptive basis scan for SPC}

The wavelet decomposition leads to sparse signals allowing one to discard many coefficients at the restoration step [8]. Given this sparsity, acquiring each coefficient is not necessary. A sampling scheme can be chosen to mainly acquire significant coefficients. Deutsch et al. [5] based their approach on a father-son relationship hinged on the tree structure of the wavelet decomposition [8]. This stands that a coefficient at the scale $j$ has 4 sons at the finer scale $j-1$. A thresholding strategy was then employed to predict the relevant coefficients. Similarly, Dai et al. [6] used a more refined thresholding strategy that outperforms Deutsch's method. To overcome the limits of an image-dependant thresholding strategy, we considered in [7] to use a non-linear approximation. To avoid the father-son relationship, we propose to couple this previous strategy with an interpolation scheme.

We base our method on the non-linear approximation of the wavelet transform that retains a number $M<<P$ of the largest coefficients. This approximation was shown to give excellent image recovery [8]. In the case of the SPC, the whole wavelet transform of the object to be imaged is not known. Therefore, we perform several non-linear approximation throughout the different decomposition levels. More precisely, our strategy consists in the following steps. First, the approximation image at scale $J$ is fully acquired, this provides $n_{A}=2^{2 L}$ coefficients with $L=R-J$. This image is considered as a low resolution image from which we create an high resolution image by increasing its size by two via bi-cubic interpolation. This high resolution image is used for significant coefficients prediction by taking its one-level wavelet transform from which a percentage $p_{J}$ of its largest detail coefficients are retained. The location of those elements are then chosen for acquisition with the SPC, leading to $n_{J}=3 \times 2^{2 L} \times p_{J}$ acquired detail coefficients at $J$. For the other decomposition levels of the wavelet transform, we proceed similarly by restoring each time the approximation image at $j$ with the inverse wavelet transform. This approximation image is then used as the low resolution image employed for prediction. Therefore, at each step, we keep

$$
n_{j}=3 \times 2^{2^{l}} \times p_{j}
$$

coefficients with $l=R-j$. We thus can control the total number of coefficients $n$ acquired for each decomposition level by modulating the set of percentages $\mathscr{P}=\left\{p_{1}, p_{2}, \ldots, p_{J}\right\}$. Using (6), it can be shown that

$$
n=2^{2 L}\left[1+3 \sum_{j=1}^{J} 4^{J-j} p_{j}\right]
$$

We define the sampling rate (SR) as $n$ over the total number of pixels $P$. One can finally recover an image from the $n$ samples using the inverse wavelet transform. 

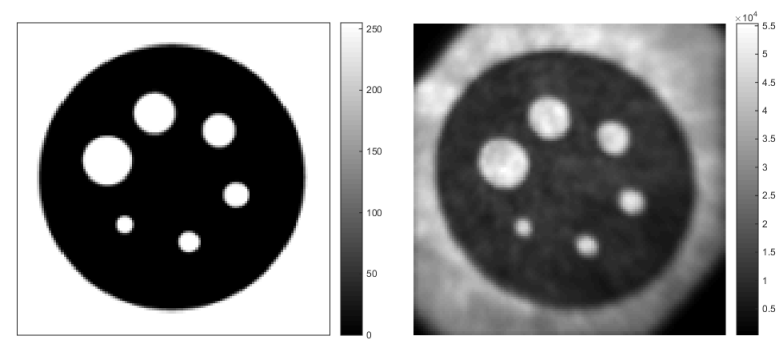

Fig. 1. Jaszczak target. $128 \times 128$ ground truth image used for simulation (left) and experimental CCD image of the printed target on a paper (right).

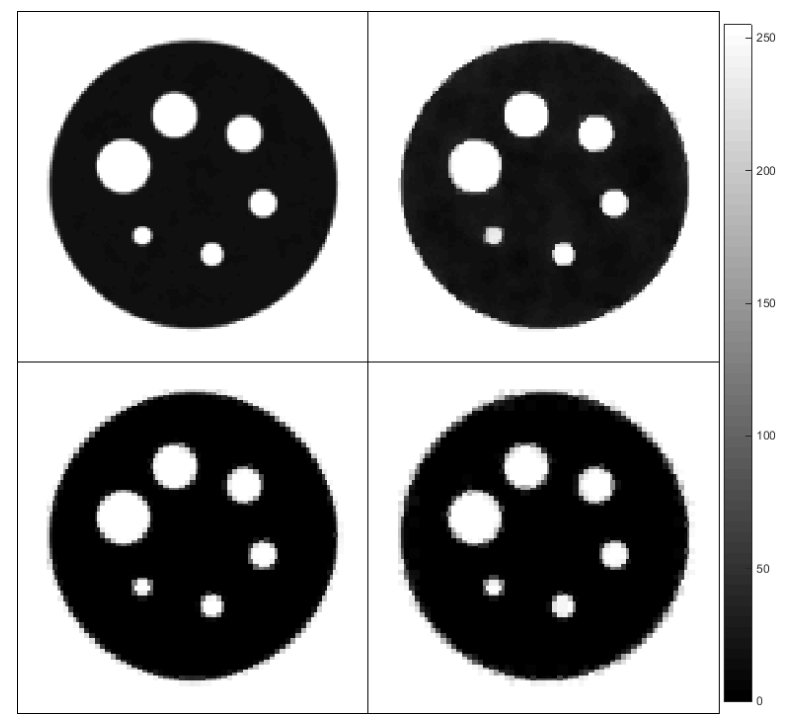

Fig. 2. Simulation of CS and ABS on the Jaszczak target. Top row: CS simulation for a SR of 20\% (left) and 10\% (right). Bottom row: AS simulation for a SR of $20 \%$ and $10 \%$. Table 1 presents the PSNRs associated with these results.

\section{RESULTS}

For CS simulation, instead of the $\ell_{1}$-minimization in (4), we directly reconstructed the image f from the measurements y using Total Variation (TV) minimization via TVAL3 [9]. This is close to performing $\ell_{1}$-minimization in the wavelet domain [10] and allow for much faster image restoration. Anisotropic TV with positivity gave the best results for the images presented here. For our method ABS, Haar patterns were considered since they are well suited for the DMD's ON/OFF technology.

In Fig. 1, we present the ground truth Jaszczak target commonly used as a phantom in CT as well as the experimental image acquired by means of a 16 bit CCD camera.

Figure 2 presents simulated results on the target for our method and CS. In the case of ABS, $\mathscr{P}=\{0.011,0.69,1,1\}$ and $\mathscr{P}=\{0.01,0.22,0.76,1\}$ where used to obtain SRs of $20 \%$ and $10 \%$. Table 1 gives the quantitative results in terms

\begin{tabular}{cccc}
\hline \hline Method & SR (\%) & Time (s) & PSNR (dB) \\
\hline \hline \multirow{2}{*}{ CS } & 20 & 15.934 & 24.16 \\
& 10 & 10.614 & 22.90 \\
\hline \multirow{2}{*}{ ABS } & 20 & 0.008 & 26.85 \\
& 10 & 0.006 & 25.05 \\
\hline \hline
\end{tabular}

Table 1. Time and PSNRs associated with the results of Fig. 2. Time takes into account the image restoration for TV and prediction+restoration for ABS. PSNRs are given with respect to the ground truth image in Fig. 1.

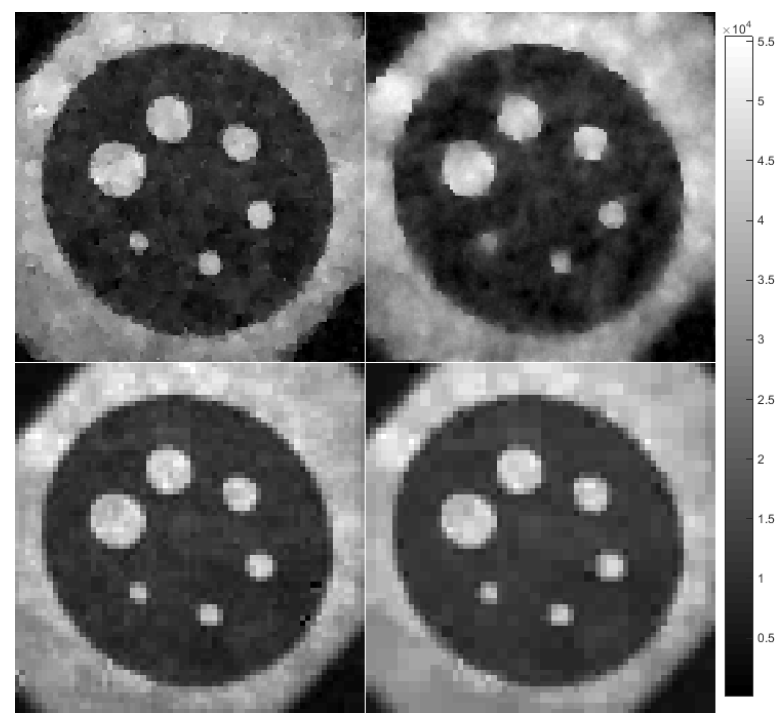

Fig. 3. Acquisition of the Jaszczak target using the SPC and images restored with CS or ABS. Top row: CS restoration for a SR of $20 \%$ (left) and $10 \%$ (right) with a PSNR of $19.70 \mathrm{~dB}$ and $19.18 \mathrm{~dB}$. Bottom row: ABS restoration for a SR of $20 \%$ (left) and $10 \%$ (right) with a PSNR of $20.90 \mathrm{~dB}$ and 20.60 dB. PSNRs are given with respect to the CCD image in Fig. 1 after registration.

of peak signal to noise ratio (PSNR) and computing time for both SRs.

In Fig. 3, we present the same results as the simulated ones but for real acquisitions using the SPC with $128 \times 128$ pixels patterns. The experimental setup was composed of a laser source operating at $650 \mathrm{~nm}$ wavelength for uniform illumination of the target. A $1024 \times 768$ DMD was exploited to spatially modulate the image. The light reflected from the DMD is focused by means of a lens on a single pixel photomultiplier detector. The target was printed on a paper with a diameter of $22 \mathrm{~mm}$.

Fluorescence imaging being a target application, we tested our acquisition strategy on an image of a mouse injected with a fluorescence dye. Results are shown in Fig. 4 for a SR of $10 \%$ and using Le Gall's wavelet (CDF 5/3). 

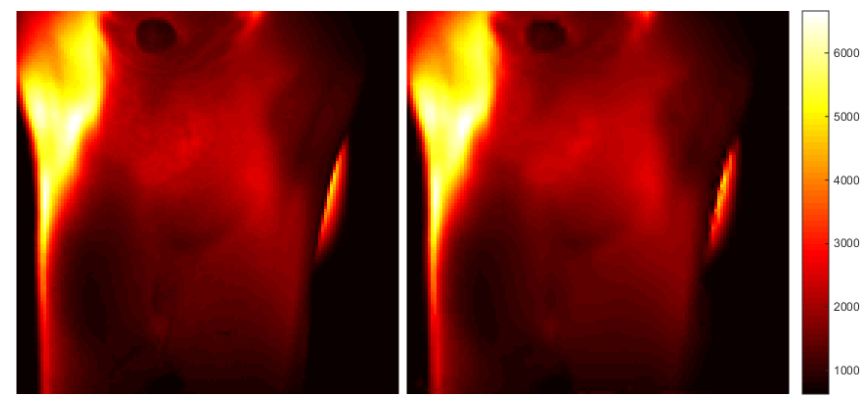

Fig. 4. Simulation of our acquisition strategy on a $128 \times 128$ image of a mouse injected with a fluorescence dye (left). Image restored for a SR of $10 \%$ using our strategy with Le Gall's wavelet (right). A PSNR of $41.75 \mathrm{~dB}$ was reached.

\section{DISCUSSION}

As can be seen in Fig. 2, visually, both the CS and ABS methods show very efficient image restoration on the phantom. For both methods, most of the errors are concentrated on the edges. By looking at the associated PSNRs given in table 1, we can see that our technique performs better. An other advantage of our method is that the restoration of the image is straightforward leading to almost negligible computation time. The many parameters of the TV algorithm are also critical to the quality of the restored image. In our technique, only the wavelet and the set of percentages have to be chosen. Those latter can be tuned according to the type of object to image. For instance, a high frequency image will need a high $p_{1}$ value (numerous details) whereas for a low frequency image, it can be set to 0 .

Similar observations can be made for the real acquisitions of Fig. 3. In our optical setup configuration, we can image an array of about $27 \times 27 \mathrm{~mm}^{2}$ and obtained a pixel pitch of 210 $\mu \mathrm{m}$ using $128 \times 128$ pixels patterns. This pixel pitch can be easily improved by increasing the size of the patterns and/or changing the focal distance of the objective lens.

Looking at the results of Fig. 4, we can see that our method shows great flexibility in the sense that the same set of percentages works well for this image and the Jaszczak target despite their clear difference. Moreover, today's DMD offer the possibility to load grey levels patterns, allowing one to chose the wavelet in our technique according to the object. For example, Le Gall biorthogonal wavelet has been successfully used in acquisition although quantization of the patterns is needed. This wavelet offers higher compression rate than Haar's wavelet.

\section{CONCLUSION}

We presented in this paper a new strategy to acquire images with a SPC. Compared to the CS technique, we obtain similar results in a shorter time by avoiding the $\ell_{1}$-minimization. We also have a small number of parameters to set with straightforward image restoration. Simulations and real acquisitions with the proposed methodology show both good visual and numerical results. In future work, we plan to use this optical setup for fluorescence lifetime imaging of biological tissues.

\section{ACKNOWLEDGEMENTS}

This work is funded by the Universite Franco-Italienne and was performed within the framework of the LABEX PRIMES, ANR-11-LABX-0063/ANR-11-IDEX-0007. It was also supported by Cariplo Foundation under Grant N. 20130615. LASERLAB-EUROPE (grant agreement no. 284464, EC's Seventh Framework Programme). EEA ED160 doctoral school is thanked for its travel grant for F. Rousset.

\section{REFERENCES}

[1] M.F. Duarte, M.A. Davenport, D. Takhar, J.N. Laska, Ting Sun, K.F. Kelly, and R.G. Baraniuk, "Single-pixel imaging via compressive sampling," Signal Processing Magazine, IEEE, vol. 25, no. 2, pp. 83-91, March 2008.

[2] N. Ducros, A. Bassi, G. Valentini, G. Canti, S. Arridge, and C. D'Andrea, "Fluorescence molecular tomography of an animal model using structured light rotating view acquisition," Journal of Biomedical Optics, vol. 18, no. 2, pp. 020503020503, 2013.

[3] D. Takhar, J. N. Laska, M. B. Wakin, M. F. Duarte, D. Baron, S. Sarvotham, K. F. Kelly, and R. G. Baraniuk, "A new compressive imaging camera architecture using optical-domain compression," in in Proc. of Computational Imaging IV at SPIE Electronic Imaging, 2006, pp. 43-52.

[4] D. L. Donoho, “Compressed sensing," IEEE Trans. Inform. Theory, vol. 52, pp. 1289-1306, 2006.

[5] S. Deutsch, A. Averbush, and S. Dekel, "Adaptive compressed image sensing based on wavelet modeling and direct sampling," in SAMPTA'09, Laurent Fesquet and Bruno Torrésani, Ed., Marseille, France, May 2009, p. General session.

[6] H. Dai, G. Gu, W. He, F. Liao, J. Zhuang, X. Liu, and Q. Chen, "Adaptive compressed sampling based on extended wavelet trees," Appl. Opt., vol. 53, no. 29, pp. 6619-6628, Oct 2014.

[7] F. Rousset, N. Ducros, C. D'Andrea, and F. Peyrin, "Single pixel camera: An acquisition strategy based on the non-linear wavelet approximation," in Engineering in Medicine and Biology Society (EMBC), 2015 37th Annual International Conference of the IEEE, Aug 2015, pp. 6240-6243.

[8] S. Mallat, A Wavelet Tour of Signal Processing, Third Edition: The Sparse Way, Academic Press, 3rd edition, Dec. 2008.

[9] C. Li, An efficient algorithm for total variation regularization with applications to the single pixel camera and compressive sensing, Ph.D. thesis, Rice University, 2009.

[10] E.J. Candes and T. Tao, "Near-optimal signal recovery from random projections: Universal encoding strategies?," Information Theory, IEEE Transactions on, vol. 52, no. 12, pp. 54065425, Dec 2006. 